\title{
FE analyses and tests in support of Wendelstein 7-X trim coil development
}

\author{
Stefan Freundt*, Andrzej Dudek, Matthias Köppen, Victor Bykov, Kostantin Egorov, \\ Joris Fellinger, Konrad Riße
}

Max Planck Institute for Plasma Physics, EURATOM Association, Wendelsteinstr. 1, 17491 Greifswald, Germany

\begin{abstract}
The paper presents an overview of the Finite Element (FE) analyses on the trim coils (TCs) and tests performed at IPP, and focuses on some specific aspects of the TC assembly. Global FE models (GM) of two types of TCs predicted the coil deformations and reaction forces on the supports. Dead weight, temperature rise during operation up to $80^{\circ} \mathrm{C}$, electromagnetic loads of 19 field configurations, and outer vessel (OV) deformations have been taken into account. In addition, local models were created for the critical winding pack (WP) regions as well as for each support clamp, and were analyzed with boundary conditions determined by the GM. The detailed WP sub-models were developed to verify the insulation strength against local stresses. The simulations were supported by various mechanical tests under static and cyclic loads. Aim of these experiments was to determine the time dependent behavior of the silicone rubber pads which are used as interfaces between TC and supports, and to check and optimize the assembly tools as well as procedures for the TCs and their supports.
\end{abstract}

Keywords: Wendelstein 7-X, magnetic field, trim coils, finite element analyses, silicone rubber

\section{Introduction}

The Wendelstein 7-X (W7-X) stellarator is under construction at the Max Planck Institute for Plasma Physics (IPP) in Greifswald, Germany [1]. Its superconducting magnet system is designed to generate a fivefold symmetric electromagnetic (EM) field. However, due to manufacture and assembly tolerances, small field asymmetries are expected to occur [2]. In order to compensate such disturbances and to fine tune the magnetic field, a set of five water cooled copper coils, so called trim coils (TCs), have been designed in collaboration with Princeton Plasma Physics Laboratory (PPPL) [3]. The latter also provide the coils which are now ready for assembly.

The TCs are externally mounted on the outer vessel
$(\mathrm{OV})$, using supports as shown in figure 1. There is one trim coil per W7-X module. Due to restricted space, the trim coil in module 2 (type B) differs from all other trim coils (type A) [3].

\section{Layout and functional requirements for the TC supports}

Each of the five trim coils is held by either four (type B) or five (type A) supports. Every support consists of three parts: a hollow cantilevered beam which is welded to the OV, a steel clamp holding the coil in the designed position and a steel adapter connecting the beam and the clamp, see figure 1 .
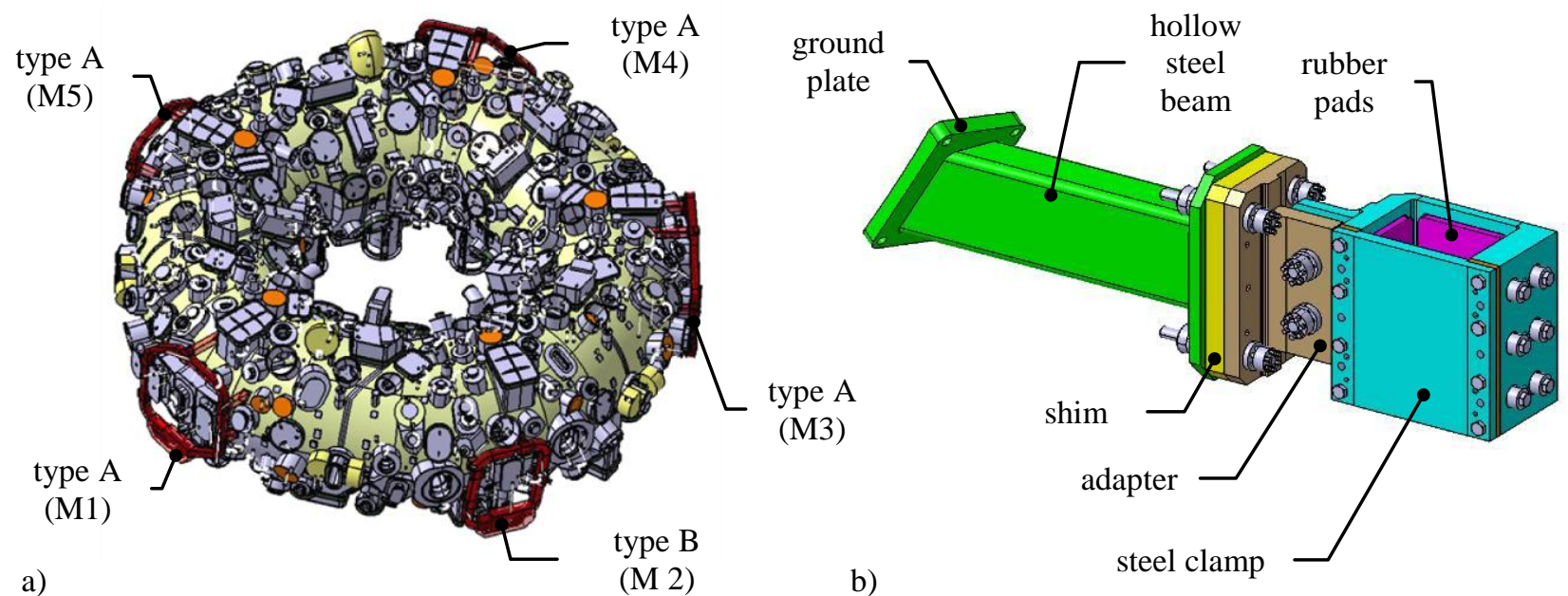

Fig. 1: Trim coils and their supports

a) Location of trim coils type A and type B in modules (M) 1 to 5 of W7-X, b) Trim coil supports 
The TC support structure is subjected to the following loads: the dead weight of the TC, EM loads, deformation of the $\mathrm{OV}$, and thermal expansion of the TC during operation. The dimensions of the support components are tightly limited due to space restrictions. Additionally, relative to their nominal position the support structure must allow $\pm 22 \mathrm{~mm}$ deviations of the OV surface, $\pm 5 \mathrm{~mm}$ tolerances of $\mathrm{TC}$ manufacturing, and $\pm 3 \mathrm{~mm}$ for TC adjustment during assembly. The TC displacements during operation are specified to remain within $\pm 5 \mathrm{~mm}$.

In order to compensate for both the OV manufacturing tolerances and assembly misalignments, the steel adapter comprises two steel flanges which are bolted together. The main adjustment is achieved with customized, 3D milled shims between the flanges. Slotted holes in the flanges give additional possibilities for fine tuning, see figure $1 b$.

To accommodate local deviations of the TC surface and displacements which are expected during operation, three preloaded silicone rubber pads are mounted at each side of a clamp, see figure $1 \mathrm{~b}$. They provide a flexible interface between the clamp and the TC and at the same time eliminate the risk of TC insulation damage during the planned 60,000 cycles of operation.

The rubber pads are placed into recesses in the clamp to provide a form lock and to prevent sliding out of the clamp during operation, compare figure 5. To increase the coefficient of friction (COF) between the silicone rubber pads and the TC to a value above 0.3 , the corresponding surfaces of the TCs are coated with a special layer of corundum particles. Between the rubber pads and the shoulders of the recesses a $1 \mathrm{~mm}$ gap is foreseen to allow the pads to expand during the preload procedure and to limit their stiffness.

\section{Results of FE analyses}

Several finite element analyses (FEAs) were carried out in order to check and improve the design of the TC supports, to predict global deformations, and benchmark FE calculations performed by PPPL.

\section{Global FE analyses}

The objective of the global FEAs was to investigate the global behavior of the TCs under dead weight, estimated temperature distribution, EM loads for 19 reference field configurations, and loads from OV deformation (e.g. due to atmospheric pressure and dead weight).

In the global FE model orthotropic properties of the TC insulation were assumed. The silicone rubber pads inside the support clamps were modeled using linear elastic material properties and real frictional contact between the rubber pads and the TC.

Furthermore, global FE model analyses have been performed to investigate the influences of the contact settings and the material parameters of the silicon rubber pads. Two extreme cases were considered. One calculation investigated the maximum displacement of the TCs in case of frictional contact in combination with a Young's modulus of $5 \mathrm{MPa}$ for the rubber. A second calculation looked at maximum loads on the supports in case of a bonded multi point constraint (MPC) contact and a Young's modulus of $150 \mathrm{MPa}$ for the rubber, see figure 2 .

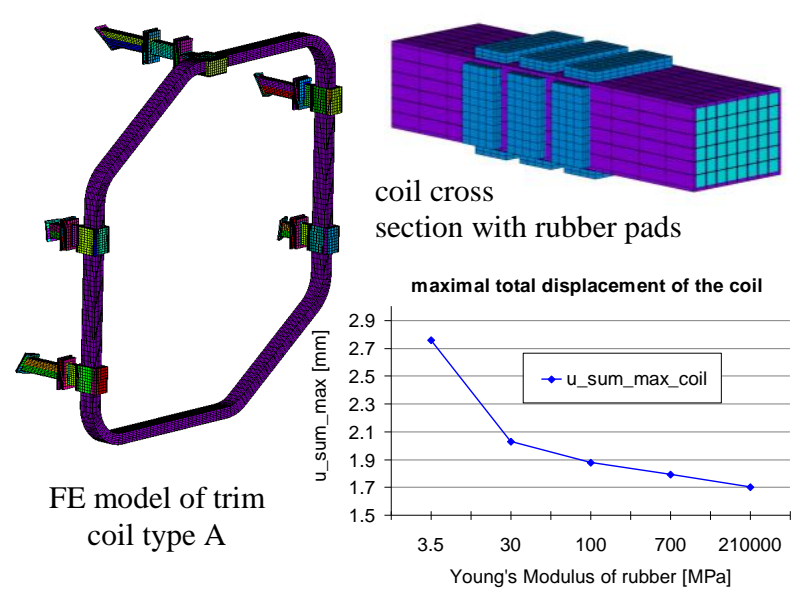

Fig. 2: Influence of the silicone rubber's stiffness on the TC displacements, here given for TC type A

Both of the above cases were simulated for all 19 EM reference field configurations. The electromagnetic force densities - needed as input for the FEAs - were calculated with an in-house code numerically solving the volume integral of the cross product of current densities and magnetic flux densities over the current carrying geometries. Figure 3 shows the distribution of the force densities along the circumference of TC type A for 10 different field configurations.

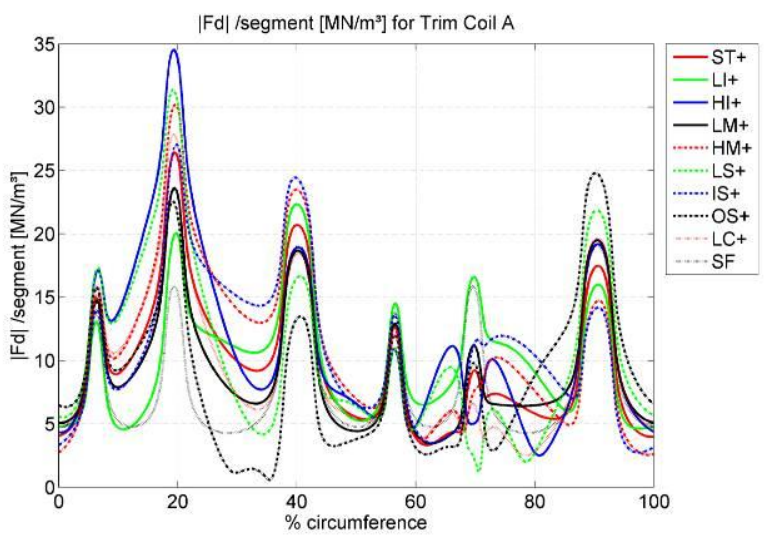

Fig. 3: Force densities along the circumference of TC type A for 10 EM field configurations

The performed global FE analyses have shown

- that the calculated TC displacements are within the required envelope of $\pm 5 \mathrm{~mm}$,

- a good agreement of the results with the PPPL FEAs regarding displacements and reaction loads in the supports $(<10 \%$ deviation $)$.

Another analysis of the results showed that approximately $40 \%$ of the coil displacement results from EM loads, an equally large part is a consequence of the TC's thermal expansion, and the remaining $20 \%$ are due to gravity and $\mathrm{OV}$ deformation. 
The resulting reaction forces and moments which act on the TC supports and on the OV were later on used as inputs for subsequent FEAs. These analyses investigated the clamps and support components in detail (see the following paragraphs). Load parameters for a series of tests on the silicon rubber pads also relied on the above results.

\section{Local winding pack analyses}

Detailed stress and strain analyses of the TC winding packs (WP) have been performed for critical coil segments using a sub-modeling technique.

The objective was to determine and confirm the shear and normal stresses and the corresponding strain of the coil insulation. Three different insulation types are used per TC: turn insulation (TI, $0.4 \mathrm{~mm}$ thick), inter layer insulation (LI, $1 \mathrm{~mm}$ ) and ground insulation (GI, $4 \mathrm{~mm}$ ).

For the local analyses orthotropic insulation material properties were used. In a first step the interconnections between different model parts have been realized with the help of bonded contacts. As boundary conditions the displacements coming from the GM and the estimated temperature gradients corresponding to the chosen cooling concept of the TCs were applied. The results from this local analysis of the TC WP have shown that the stresses and strains in the GI, LI and TI exceed the allowable only at the connection between the LI and GI.

To investigate these strains and stresses more closely in a second step, a frictional contact was modeled between GI and LI in order to allow detaching at that interface. After the GI is detached from the LI the stresses and strains in the GI, LI and TI remain below the allowable limits. Cracking and delamination of the TC insulation is therefore very unlikely.

\section{Local support analyses}

Separate FE models have been created for detailed local analyses of each clamp. The models were loaded with the forces and moments extracted from the global FE analysis results. Stresses in the clamp components as well as deformation and sliding of the rubber pads in the clamp - depending on the COF - were checked. In addition, the stresses in the welds of the stiffening ribs and in the bolted connection between the clamp and the shim plate have been investigated, see figure 4 .

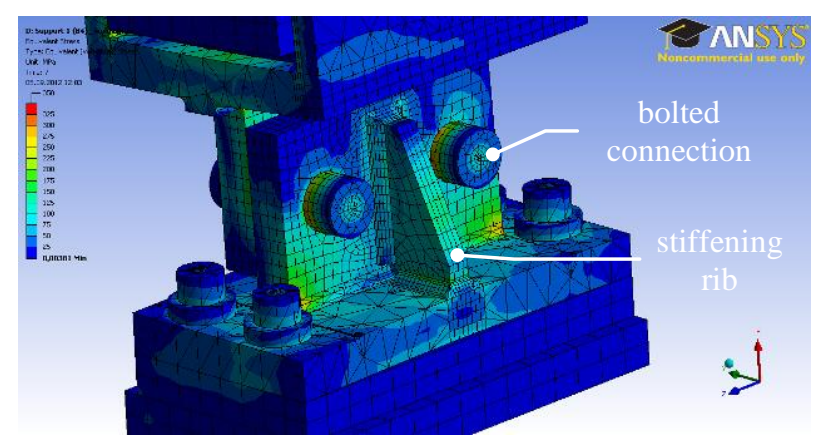

Fig. 4: Local FE model of the most loaded bolted connection of TC type B
Based on the FE results the preload required on the rubber pads and on the bolts during assembly were defined. The same results were also used to define the assembly strategy of the clamps and the preload sequence of the rubber pads.

For the most critical bolted connection between the clamp and the adapter (see figure 1b) an increased friction between the contact surfaces is required. This is achieved by machining the surfaces in defined directions (micro form locking) and high pre-stress of the bolts.

\section{Accompanying tests on rubber pads}

A series of tests has been carried out to support the design activities and FE analyses. The aim of the tests was to identify mechanical parameters of the silicon rubber pads under defined loads.

Preliminary tests had shown that a COF $>0.3$ on the contact surfaces between the TC and the silicone rubber pads is necessary to avoid sliding and to keep the coil in position under loads resulting from the FEA. To achieve this COF a special coating layer using corundum particles is applied to the TC. Accompanying tests using such layers have been performed to confirm the COF and to check the behavior of the silicone rubber pads. Additional tests were carried out to qualify the coating process and to develop the coating tools.

\section{Static tests}

A settling test and several simple compression tests were performed in order to:

- investigate and determine the relaxation and the longterm behavior of the preloaded rubber pads,

- define and confirm the preload force and deflection as a design value of the clamps.

The extrapolated results of the static settling test at room temperature (RT) and $80^{\circ} \mathrm{C}$ showed up to $45 \%$ loss of preload during the assumed life time of 15 years. This result has been confirmed in separate static compression tests using silicon rubber pads with original dimensions.

Additional tests were performed for defining the required pre-stress and deformation of the pads during assembly. In these tests the generalized compression modulus of the silicone rubber pads was determined for the given gaps between the pads and the recess in the steel plate, see figure 5 .

\section{Cyclic tests}

The cyclic tests included 1000 compression and shear cycles each, performed under RT. The loads were derived from the global FEAs. Both tests were carried out on a compression-tension test machine.

The performed tests confirmed the chosen silicone rubber pads as sufficiently stable under static and varying loads. The COF between the rubber and the corundum coating has been verified to reach at least 0.5 . Based on the tests a preload of $1.0 \mathrm{MPa}$ for the main preload direction and $0.5 \mathrm{MPa}$ for the secondary preload 
direction was chosen. In doing so, sufficient preload is guaranteed over the life time despite the relaxation. However, due to creeping and the 60000 load cycles during 15 years the preload of the rubber pads decreases by additional $20 \%$.

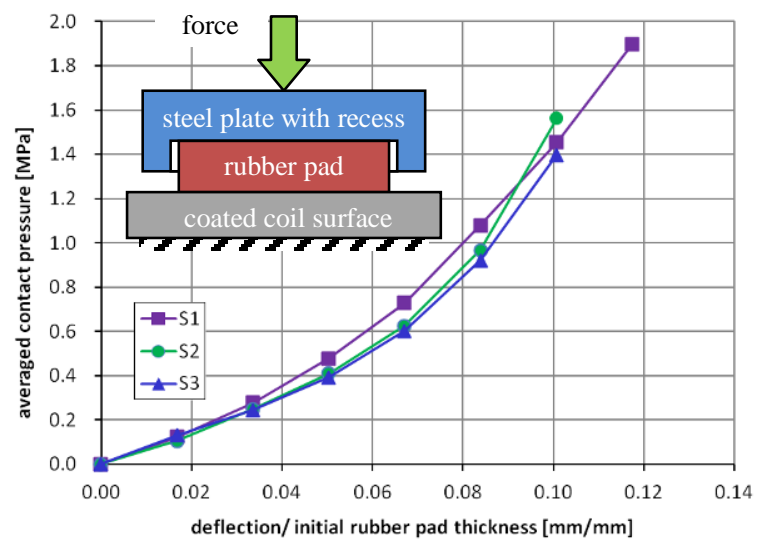

Fig. 5: Compression tests (S1 to S3) to determine the generalized compression modulus of the silicone rubber pads

\section{Selected aspects of $\mathrm{TC}$ assembly}

For TC transport and assembly a special transport frame and a lifting device were developed at IPP. In order to support the design process for these structures, FE analyses and tests were performed. These calculations especially focused on TC deformations during craning and assembly.

The FE model for the transport frame and lifting device includes the header region and the TC supports; it also takes into account the stiffness and dead weight of the TC. Temporary assembly supports with rubber pads inside have been designed to lift the TCs (figure 6). The dimensions and required preload of the assembly support rubber pads were defined based on FEA results.

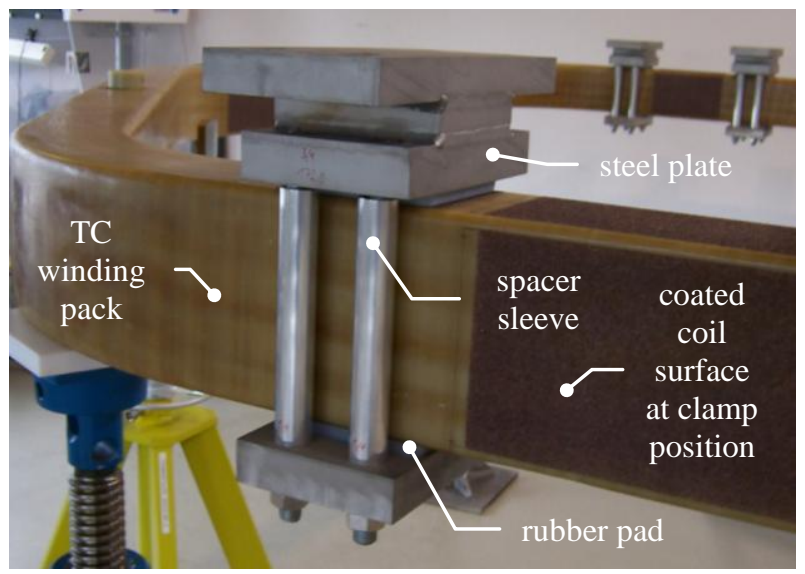

Fig. 6: Temporary assembly supports

In preparation of the final $\mathrm{TC}$ assembly a number of assembly tests were performed on an assembly mock-up to check the handling and the assembly strategy, and to derive the final assembly and maintenance procedures for the clamps.
A set of thin steel shims and washers with different thicknesses simplifies retightening of the rubber pads. When the preload is below the required limit, e.g. due to relaxation, it is foreseen to just remove some washers and preload the rubber pads again without dismounting the whole clamp. This will save time and costs during maintenance.

In addition, a special tool for checking the preload of the silicone rubber pads has been developed.

\section{Summary}

The trim coils are mostly manufactured and ready to be mounted on the outer vessel of the W7-X cryostat. They will be clamped via soft silicone rubber pads to their supports which are attached to the OV.

Finite Element (FE) analyses were performed using

- a global FE model to predict the TC deformations and reaction forces on the supports,

- a local FE sub-model of the critical WP segments to verify the insulation strength against local stresses,

- local FE models for each support clamp to simulate the behavior under loads and moments which were extracted from the global FE model.

The simulations were complemented by several mechanical tests to determine the time dependent behavior of the rubber pads under static and cyclic loads. Additional tests and FE analyses have been carried out to check and optimize the assembly tools and procedures for the TCs.

\section{Acknowledgments}

The authors would like to thank L. Kästner of AirBolting comp. for providing data and general information on rubber materials. Special thanks go to the TC team at IPP Greifswald for fruitful discussions and valuable contributions. The work of $\mathrm{R}$. Tesch of Fachhochschule Stralsund supporting the cyclic tests of the silicone rubber pads is also gratefully acknowledged.

\section{References}

[1] L. Wegener et al, Status of Wendelstein 7-X construction, Fusion Engineering and Design vol. 84, no. 2-6, pp. 106-112, June 2009

[2] J. Kißlinger and T. Andreeva, "Correction possibilities of magnetic field errors in Wendelstein $7-X^{\prime \prime}$, in Fusion Engineering and Design 74 (2005) 623-626.

[3] K. Riße et al, Design and manufacturing status of trim coils for the Wendelstein 7-X stellarator experiment, SOFT 2012 (this conference) 